Erratum

\title{
Erratum to "Verification, Validation, and Testing of Kinetic Mechanisms of Hydrogen Combustion in Fluid-Dynamic Computations"
}

\author{
Victor P. Zhukov \\ Institute of Space Propulsion, German Aerospace Center (DLR), Lampoldshausen, 74239 Hardthausen, Germany \\ Correspondence should be addressed to Victor P. Zhukov; victor.zhukov@dlr.de \\ Received 2 October 2013; Accepted 13 October 2013; Published 2 January 2014 \\ Copyright (C) 2014 Victor P. Zhukov. This is an open access article distributed under the Creative Commons Attribution License, \\ which permits unrestricted use, distribution, and reproduction in any medium, provided the original work is properly cited.
}

A typing error in Table 1 in page 5 was detected. The chemical equation of reaction $\mathrm{R} 2$ was typed incorrectly:

$\mathrm{R} 2 \mathrm{H}_{2}+\mathrm{O} \longrightarrow \mathrm{H}+\mathrm{OH}_{2}$

The correct chemical equation is

$\mathrm{R} 2 \mathrm{H}_{2}+\mathrm{O} \longrightarrow \mathrm{H}+\mathrm{OH}$

The correct complete kinetic mechanism can be found in Table 1 . 
TABLE 1: New skeletal mechanism.

\begin{tabular}{|c|c|c|c|c|c|c|}
\hline Numbers & $\begin{array}{c}\text { Reference } \\
\text { number }\end{array}$ & Reaction & $A$ & $n$ & $E_{a}$ & Reference \\
\hline 1 & $\mathrm{R} 1$ & $\mathrm{H}+\mathrm{O}_{2} \rightarrow \mathrm{OH}+\mathrm{O}$ & $1.91 e+14$ & 0.0 & 16.44 & {$[30]$} \\
\hline 2 & $\mathrm{R} 2$ & $\mathrm{H}_{2}+\mathrm{O} \rightarrow \mathrm{H}+\mathrm{OH}$ & $5.08 e+4$ & 2.67 & 6.292 & {$[30]$} \\
\hline 3 & R3 & $\mathrm{H}_{2}+\mathrm{OH} \rightarrow \mathrm{H}+\mathrm{H}_{2} \mathrm{O}$ & $2.16 e+8$ & 1.51 & 3.43 & {$[30]$} \\
\hline 4 & $\mathrm{R} 5$ & $\mathrm{H}_{2}+\mathrm{M} \leftrightarrow \mathrm{H}+\mathrm{H}+\mathrm{M}$ & $4.57 e+19$ & -1.4 & 105.1 & {$[13]$} \\
\hline 5 & R6 & $\mathrm{O}+\mathrm{O}+\mathrm{M} \leftrightarrow \mathrm{O}_{2}+\mathrm{M}$ & $6.17 e+15$ & -0.5 & 0.0 & {$[13]$} \\
\hline 6 & R7 & $\mathrm{H}+\mathrm{O}+\mathrm{M} \rightarrow \mathrm{OH}+\mathrm{M}$ & $4.72 e+18$ & -1.0 & 0.0 & {$[13]$} \\
\hline 7 & $\mathrm{R} 8$ & $\mathrm{H}+\mathrm{OH}+\mathrm{M} \rightarrow \mathrm{H}_{2} \mathrm{O}+\mathrm{M}$ & $4.5 e+22$ & -2.0 & 0.0 & {$[13]$} \\
\hline 8 & R9 & $\mathrm{H}+\mathrm{O}_{2}+\mathrm{M} \rightarrow \mathrm{HO}_{2}+\mathrm{M}$ & $6.17 e+19$ & -1.42 & 0.0 & {$[30]$} \\
\hline 9 & $\mathrm{R} 10$ & $\mathrm{H}+\mathrm{HO}_{2} \rightarrow \mathrm{H}_{2}+\mathrm{O}_{2}$ & $1.66 e+13$ & 0.0 & 0.82 & {$[13]$} \\
\hline 10 & $\mathrm{R}-10$ & $\mathrm{H}_{2}+\mathrm{O}_{2} \rightarrow \mathrm{H}+\mathrm{HO}_{2}$ & $3.68 e+13$ & 0.203 & 54.46 & {$[30]$} \\
\hline 11 & R11 & $\mathrm{H}+\mathrm{HO}_{2} \rightarrow \mathrm{OH}+\mathrm{OH}$ & $1.69 e+14$ & 0.0 & 0.87 & {$[30]$} \\
\hline 12 & R13 & $\mathrm{OH}+\mathrm{HO}_{2} \rightarrow \mathrm{H}_{2} \mathrm{O}+\mathrm{O}_{2}$ & $2.89 e+13$ & 0.0 & -0.5 & {$[13]$} \\
\hline 13 & $\mathrm{R} 15$ & $\mathrm{H}_{2} \mathrm{O}_{2}+\mathrm{M} \rightarrow \mathrm{OH}+\mathrm{OH}+\mathrm{M}$ & $1.2 e+17$ & 0.0 & 45.5 & {$[30]$} \\
\hline 14 & $\mathrm{R}-17$ & $\mathrm{H}_{2}+\mathrm{HO}_{2} \rightarrow \mathrm{H}+\mathrm{H}_{2} \mathrm{O}_{2}$ & $3.42 e+12$ & 0.202 & 27.12 & {$[30]$} \\
\hline
\end{tabular}

$k=A \cdot T^{n} \cdot \exp \left(-E_{a} / R T\right)$; units: mol, $\mathrm{cm}^{3}, \mathrm{~K}$, and kcal; thermodynamic data [5]; the reverse rate constants (R5, R6) are calculated from the forward rate constants through the equilibrium constants. 

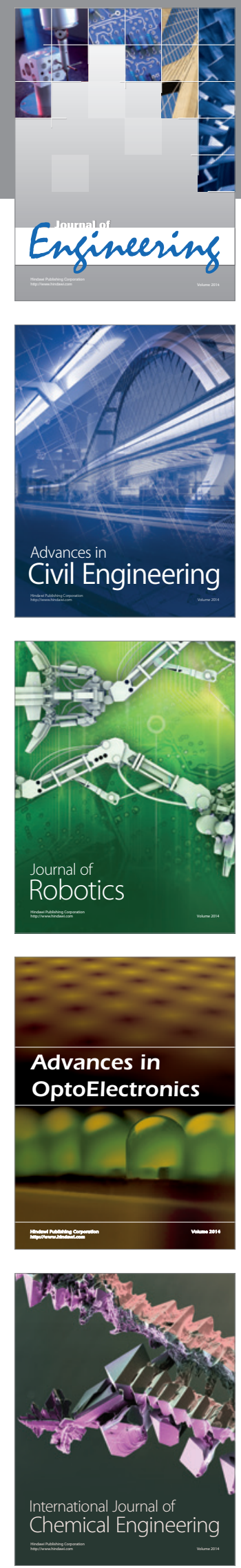

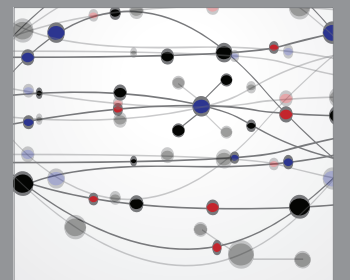

The Scientific World Journal
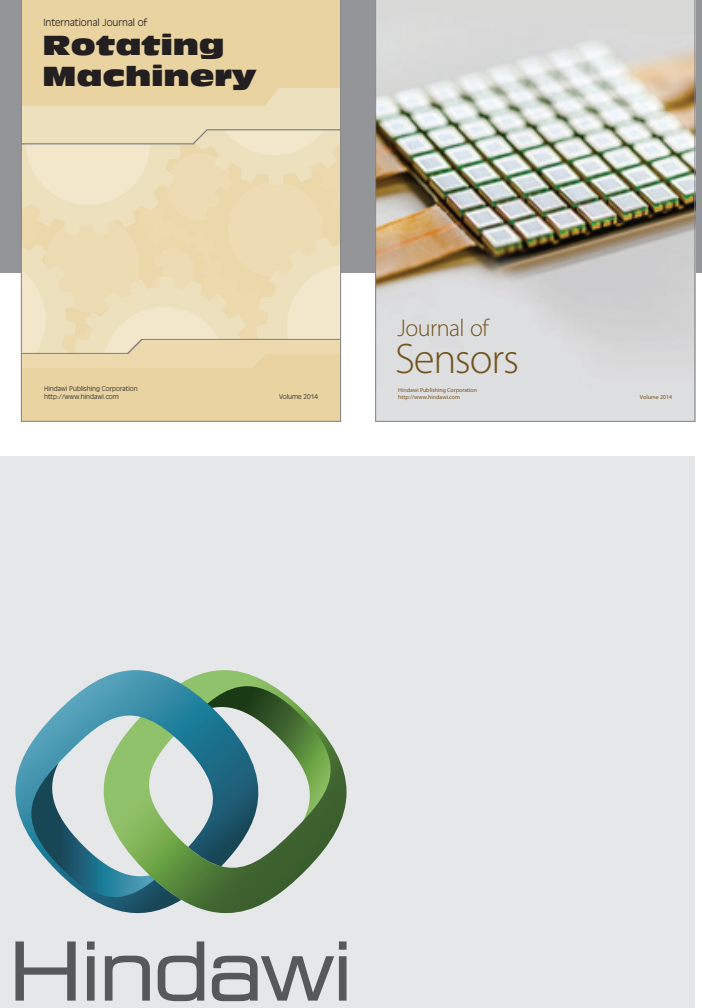

Submit your manuscripts at http://www.hindawi.com
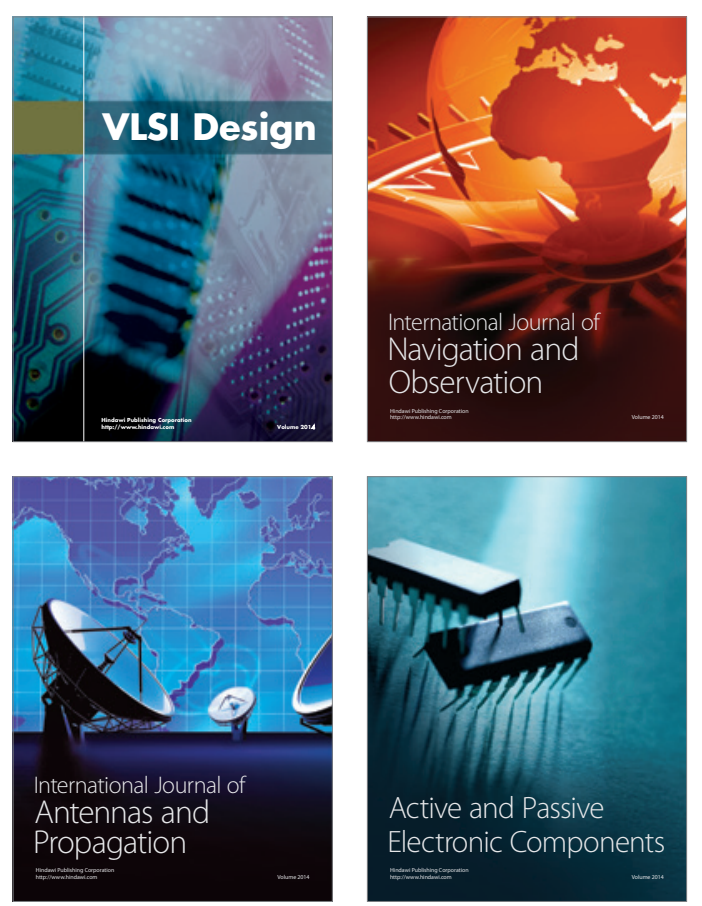
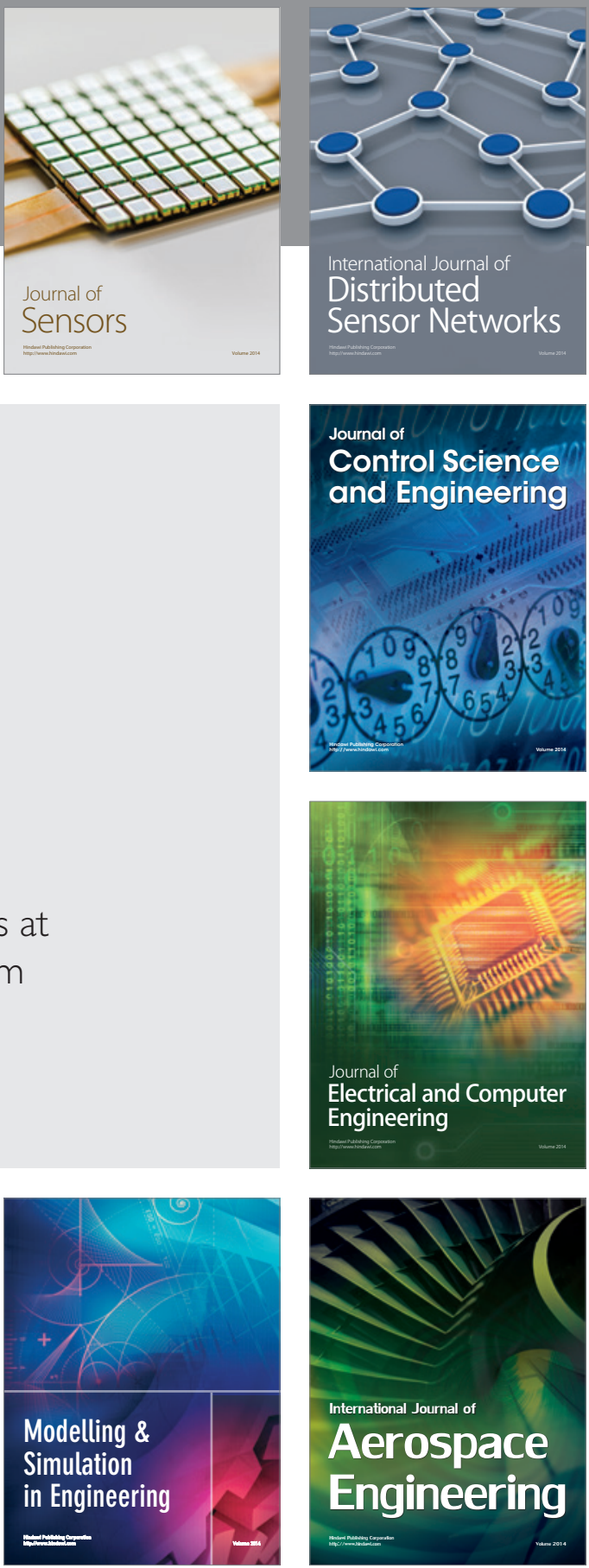

Journal of

Control Science

and Engineering
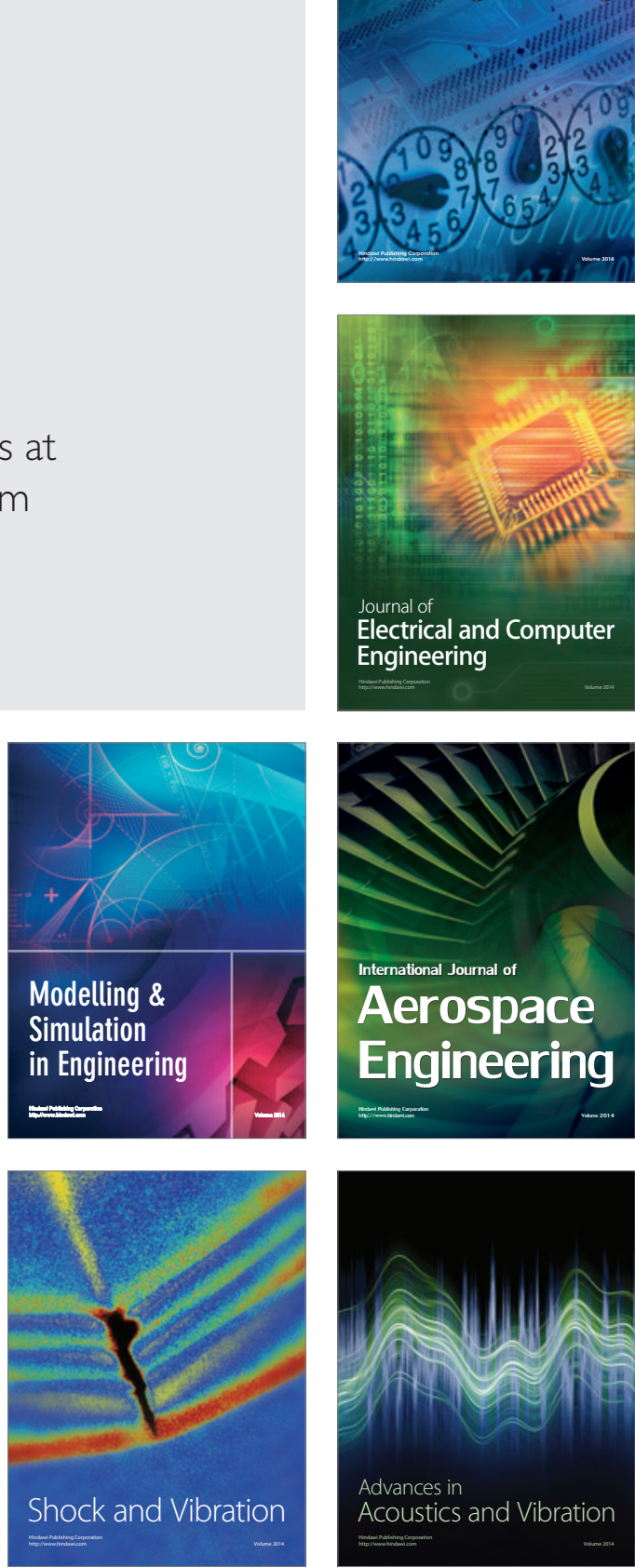